\title{
Short-term amiodarone therapy after reversion of persistent atrial fibrillation reduces recurrences at 18 months
}

\author{
Jorge Galperin ${ }^{1}$, Marcelo V. Elizari ${ }^{1}$, Ricardo Bonato ${ }^{1}$, Raul Ledesma ${ }^{2}$, \\ Manuel Vazquez Blanco ${ }^{3}$, Manuel Lago ${ }^{1}$, Pablo Spada ${ }^{4}$, \\ Jorge Sanchez ${ }^{5}$, Jorge Piasentin ${ }^{6}$, Pablo A. Chiale ${ }^{1,7}$ \\ ${ }^{1}$ Hospital Ramos Mejia, Buenos Aires, Argentina \\ ${ }^{2}$ Instituto Del Corazon, Cordoba, Argentina \\ ${ }^{3}$ Hospital De Clinicas, Buenos Aires, Argentina \\ ${ }^{4}$ Hospital De Moron, Buenos Aires, Argentina \\ ${ }^{5}$ Clinica San Rafael, Salta, Argentina \\ ${ }^{6}$ Hospital Lagomaggiore, Mendoza, Argentina \\ ${ }^{7}$ Pontificia Universidad Catolica Argentina, Buenos Aires
}

\begin{abstract}
Background: The aim of this study was to compare the outcome of 3 months vs. 18 months of amiodarone treatment after atrial fibrillation $(A F)$ conversion in patients who experienced the first episode of persistent $A F$.

Methods: We included 51 patients who experienced the first episode of persistent AF receiving amiodarone (600 mg) daily for 4-6 weeks. If AF persisted, electrical cardioversion (ECV) was performed. All patients received amiodarone (200 mg daily) for 3 months and then were randomized to amiodarone (Group I) or placebo (Group II) and followed for 15 months. The control group comprised 9 untreated patients undergoing ECV. Treatment effectiveness was evaluated using a Bayesian model.
\end{abstract}

Results: Eighteen months after AF reversion, 22 (81.5\%) patients in Group I, 13 (54.2\%) patients in Group II, and 1 (11.1\%) patient in the control group remained in sinus rhythm. No differences were found between Group I patients who required ECV and Group II patients. Sinus rhythm was preserved in all Group I patients when it was achieved during amiodarone administration. Limiting adverse effects occurred in 3 (11.1\%) patients in Group I.

Conclusions: In patients regaining sinus rhythm after the first episode of persistent $A F$, a 3-month amiodarone treatment after reversion is a reasonable option for rhythm control. (Cardiol J 2014; 21, 4: 397-404)

Key words: amiodarone, atrial fibrillation, arrhythmia-sinus rhythm maintenance

Address for correspondence: Dr Jorge Galperin, Hospital Ramos Mejia, Buenos Aires, Lafinur 2932, Buenos Aires-1425, Argentina, e-mail: jorge.galperin@gmail.com

Received: 31.07.2013 Accepted: 08.11.2013 


\section{Introduction}

Atrial fibrillation (AF) is the most common sustained tachyarrhythmia found in the adult population. It occurs in about $2-3 \%$ of the general population over 60 years of age, and in nearly $10 \%$ of individuals above 80 years. Long-term prevention of arrhythmia relapses reduces morbidity and improves quality of life and exercise capacity $[1,2]$. Therefore, restoration of normal sinus rhythm (SR) is usually attempted in patients with persistent AF, either by pharmacological or electrical means [3].

It is established that in the absence of antiarrhythmic therapy, successful cardioversion of the first episode of persistent AF is followed by a high rate of relapses ( $>75 \%$ at 1 year). This can be prevented in a substantial percentage of patients by using long-term amiodarone therapy [4-6]. However, amiodarone treatment is associated with significant side effects, including hyperthyroidism or hypothyroidism, pulmonary and hepatic toxicity, and neurological symptoms, which may preclude the benefits of this approach $[7,8]$. These side effects are much more frequent after long-term treatments. We hypothesized that maintenance of SR with amiodarone after reversion of the first episode of persistent AF for a period of only 3 months, may attenuate, or even reverse, arrhythmia-induced atrial remodeling [9-11]. This, in turn, could prevent AF recurrences and eliminate the need for long-term amiodarone therapy and the associated side effects. Thus, the aim of this study was to evaluate comparatively the rate of $\mathrm{AF}$ recurrences and amiodarone-related major adverse events during an 18-month follow-up period. Amiodarone was administered to patients with first-episode persistent AF (lasting no longer than 1 year after onset) for a period of only 3 months, or throughout the 18-month period.

\section{Methods}

\section{Patient population and study protocol}

Patients with persistent AF lasting more than 1 week and less than 1 year were included in this study. During the run-in period, persistent arrhythmia was documented with at least 3 resting electrocardiography (ECG) recordings, performed at different intervals, and by a 24-h ECG Holter monitor in the week prior to the initiation of the study. Exclusion criteria included: paroxysmal $\mathrm{AF}$; atrial flutter; acute myocardial infarction in the preceding 6 months; PR interval $>0.28 \mathrm{~s}$ in ECGs recorded prior to AF onset; second or third degree atrioventricular block in ECG tracings obtained before $\mathrm{AF}$ occurrence (unless a permanent pacemaker was implanted), spontaneous heart rate $<50 \mathrm{bpm}$; evidence of sinus node dysfunction without implanted pacemaker; QTc interval $\geq 0.50 \mathrm{~s}$; thyroid dysfunction; abnormal liver function tests; chronic lung disease; pregnancy; anteroposterior left atrium diameter $\geq 60 \mathrm{~mm}$; severe mitral stenosis or insufficiency; severe tricuspid regurgitation; elevated pulmonary artery systolic pressure; contraindications for anticoagulation; co-morbidities conditioning short-term prognosis; inability to attend follow-up sessions for any reason; and denied consent. The first documented recurrence of $\mathrm{AF}$ was considered the end follow-up point for every patient.

All participants underwent a baseline cardiovascular evaluation that consisted of a complete clinical examination, ECG at rest, 24-h ECG Holter recording, chest radiography, 2-dimensional (2-D) echocardiogram with Doppler, and standard laboratory tests, including thyroid hormone concentrations. All patients, effectively anticoagulated for at least 4 weeks (international normalization ratio: $2.0-3.0$ ), were loaded with oral amiodarone (600 mg/daily) for 4-6 weeks. Patients who regained SR during this period were subsequently treated with amiodarone $(200 \mathrm{mg} /$ daily $)$ for 3 months. In those individuals in whom AF persisted, electrical cardioversion (ECV) was performed under general anesthesia with an initial direct current monophasic shock of 200 joules and additional shocks of 360 joules when the first shock was ineffective. After successful ECV, amiodarone was administered at daily doses of $200 \mathrm{mg}$ for 3 months.

Patients were subsequently randomized in a double-blind fashion to receive amiodarone, $200 \mathrm{mg}$ daily (Group I) or placebo (Group II). Both groups were followed for 15 months. A third group that did not receive any treatment (control group) and comprised 9 patients undergoing ECV without antiarrhythmic treatment before and after conversion to SR. Follow-up evaluation consisted of a clinical examination and an ECG at rest every 30 days. A more complete assessment, including chest X-ray, 24-h Holter ECG, 2-D echocardiogram with Doppler, and standard laboratory tests including thyroid function tests, was performed at 6 -month intervals, or at any time when cardiovascular or non-cardiovascular symptoms occurred. When appropriate, anticoagulation was interrupted after at least 1 month of permanent SR.

Seven centers in Argentina engaged in this trial complied with the principles of the Declaration 
Table 1. Baseline clinical characteristics of the patients.

\begin{tabular}{|c|c|c|c|}
\hline & Amiodarone ( $n=27$ ) & Placebo $(n=24)$ & Total $(n=51)$ \\
\hline Age $(\bar{x})$ & $50-83(69.3)$ & $50-80(67.2)$ & $50-83(67.9)$ \\
\hline Sex (male) & 20 & 18 & 38 \\
\hline Dilated cardiomyopathy & 4 & 5 & 9 \\
\hline Coronary heart disease & 4 & 3 & 7 \\
\hline Hypertension & 17 & 12 & 29 \\
\hline Lone AF & 4 & 3 & 7 \\
\hline AF duration indeterminate & 4 & 6 & 10 \\
\hline $\begin{array}{l}\text { (> } 1 \text { month) } \\
1-12 \text { month } \\
(\bar{x}=\text { month })\end{array}$ & $23(\bar{x}=4.0)$ & $18(\bar{x}=5.3)$ & $41(\bar{x}=4.6)$ \\
\hline Echocardiography & Amiodarone & Placebo & Total $(n=51)$ \\
\hline LV diastolic diameter [mm] & $34-62(\bar{x}=50.9)$ & $40-70(\bar{x}=52.7)$ & $34-70(\bar{x}=51.7)$ \\
\hline LV systolic diameter [mm] & $19-52(\bar{x}=33.2)$ & $22-56(\bar{x}=35.4)$ & $19-56(\bar{x}=34.3)$ \\
\hline Interventricular septum [mm] & $8-15(\bar{x}=11.3)$ & $7-16(\bar{x}=11.3)$ & $7-16(\bar{x}=11.3)$ \\
\hline Posterior wall [mm] & $7-13(\bar{x}=10.0)$ & $7-14(\bar{x}=10.2)$ & $7-14(\bar{x}=10.1)$ \\
\hline Left atrium anteroposterior diameter [mm] & $34-59(\bar{x}=47.3)$ & $35-63(\bar{x}=46.6)$ & $34-63(\bar{x}=46.8)$ \\
\hline
\end{tabular}

$\mathrm{AF}$ - atrial fibrillation; LV - left ventricular

of Human Rights of Helsinki, and the study protocol was approved by the Ethical Board of each center. All patients were informed about the purpose of the study and they provided written informed consent before the enrollment.

\section{Statistical analysis}

The antiarrhythmic effect of amiodarone treatment is expressed as $\emptyset ; \emptyset 1=\pi \alpha-\pi \mathrm{p}$ and $\varnothing 2=\pi \mathrm{p}-\pi \mathrm{c}$, where $\pi \alpha$ is the proportion of AF recurrence in Group I, $\pi$ p represents the proportion of recurrence in Group II, and $\pi \mathrm{c}$ is the proportion of AF recurrence in the control group.

The inference with regard to $\varnothing$ was made by means of a Bayesian perspective on the estimation of the posterior probability density (ppd) of $\varnothing$. This was obtained for the whole group and for the subgroups defined by an interest factor (conditional group) and estimated by means of a Monte Carlo simulation assuming a previous non-informative distribution of both parameters. The information about $\varnothing$ contained in ppd is resumed by the median and a $95 \%$ credibility interval of equiprobability at the extremes.

\section{Results}

Table 1 summarizes the baseline clinical characteristics of patients assigned to the 2 arms of the study. The 2 groups were well matched with respect to baseline clinical findings. The predominant underlying disease was systemic hypertension, and most of the patients had evidence of structural heart disease. Group I included 27 patients, Group II 24 patients, and the control group included 9 patients who underwent ECV without antiarrhythmic treatment before and after conversion to SR.

In total, out of 51 patients, 30 (58.8\%), who received loading amiodarone treatment, regained normal SR during the initial 4-6 weeks of therapy, while in the remaining 21 patients, this was accomplished using ECV. SR was restored during the initial period of amiodarone administration in $16(59.26 \%)$ patients of Group I and in $14(58.33 \%)$ patients of Group II. Furthermore, 11 patients in Group I and 10 patients in Group II did require ECV, as shown in Figure 1.

Figure 2 depicts the Kaplan-Meier survival estimates of SR maintenance in the 3 study groups, while Figure 3 displays the Kaplan-Meier survival estimates of SR persistence during long-term and short-term amiodarone treatment, according to the $\mathrm{AF}$ reversion mode during the initial period of amiodarone therapy. After the 18-month follow-up period, normal SR was still present in $22(81.5 \%)$ patients of Group I, in 13 (54.2\%) patients of Group II, and in only $1(11.1 \%)$ patient of the control group. The rate of SR maintenance at the end of the follow-up period was similar in patients receiving amiodarone for 3 months and those on long-term treatment (Group I) that had required ECV. All patients of Group I in whom AF was converted 


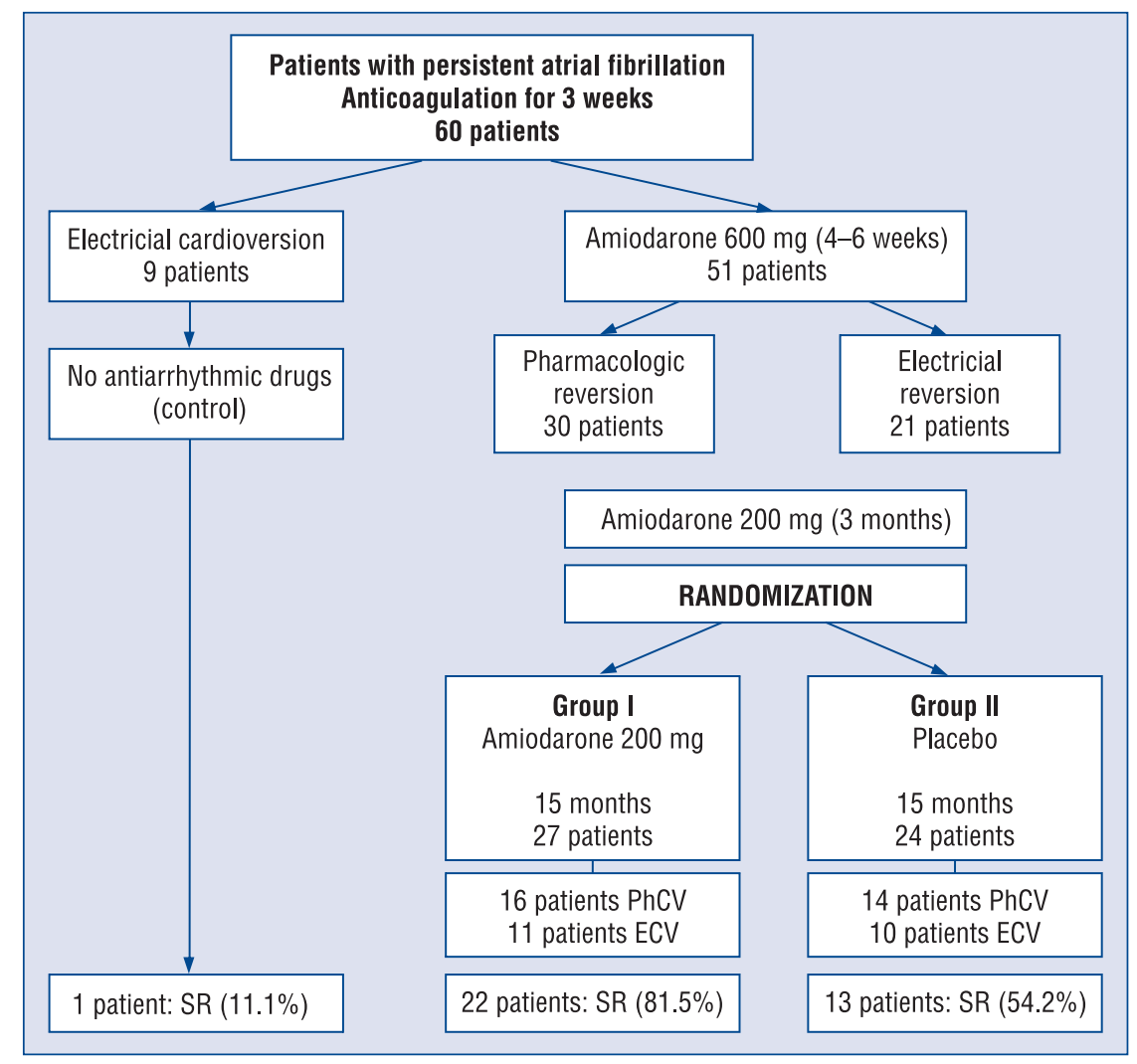

Figure 1. Study protocol in patients with first episode of persistent atrial fibrillation; ECV - electrical cardioversion; PhCV - pharmacologic cardioversion; SR - sinus rhythm.

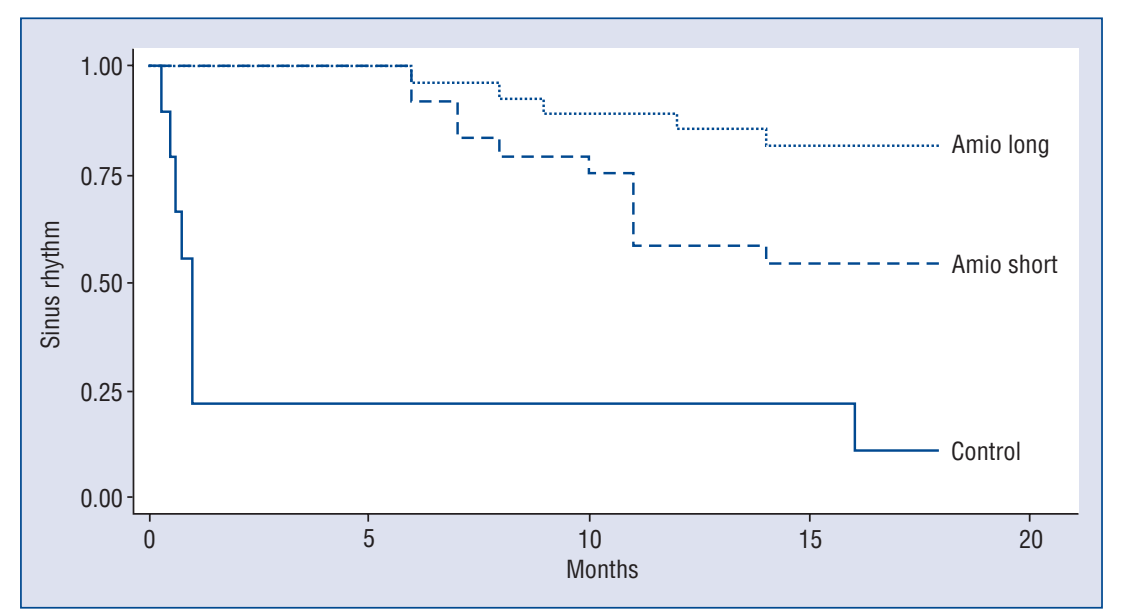

Figure 2. Kaplan-Meier survival estimates of sinus rhythm maintenance during long-term and after short-term amiodarone (amio) therapy.

pharmacologically during the initial 4-6 weeks of amiodarone therapy maintained normal SR at the end of follow-up. In marked contrast, in the same group, this occurred in only $54.5 \%$ of patients who required ECV. In Group II patients, long-term maintenance of normal SR was observed in $50 \%$ of patients who recovered SR pharmacologically during the initial 4-6 weeks of amiodarone treatment and in $60 \%$ of those who underwent ECV. Significant adverse effects were seen in $3(11.1 \%)$ patients receiving long-term amiodarone treatment ( 2 patients developed hyperthyroidism and 1 pa- 


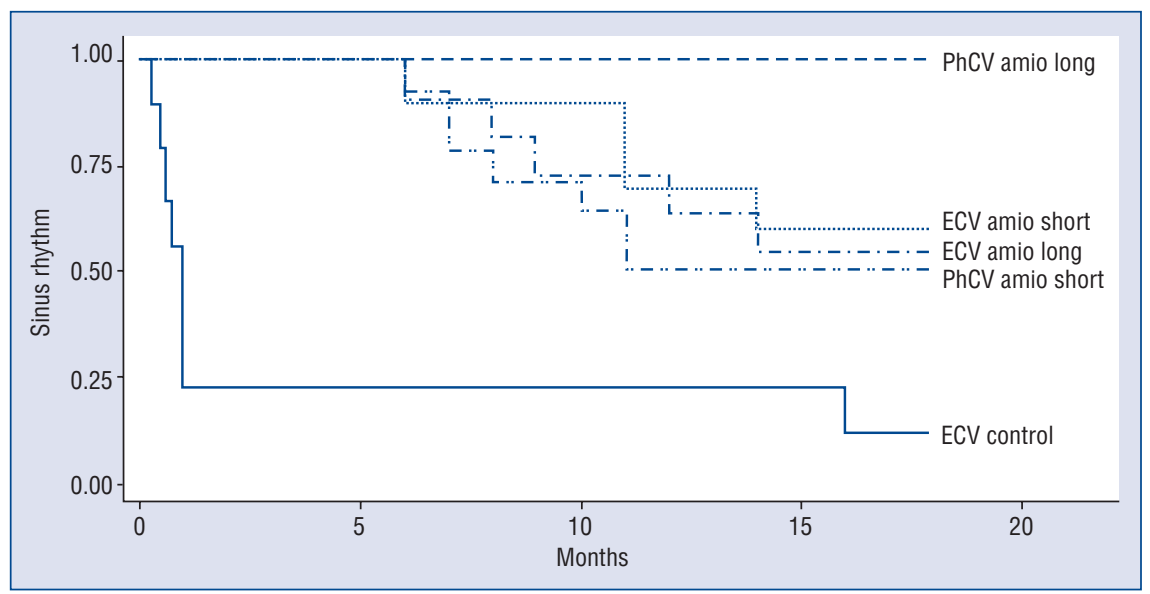

Figure 3. Kaplan-Meier survival estimates of sinus rhythm persistence according to atrial fibrillation reversion mode; amio - amiodarone; ECV - electrical cardioversion; PhCV - pharmacologic cardioversion.

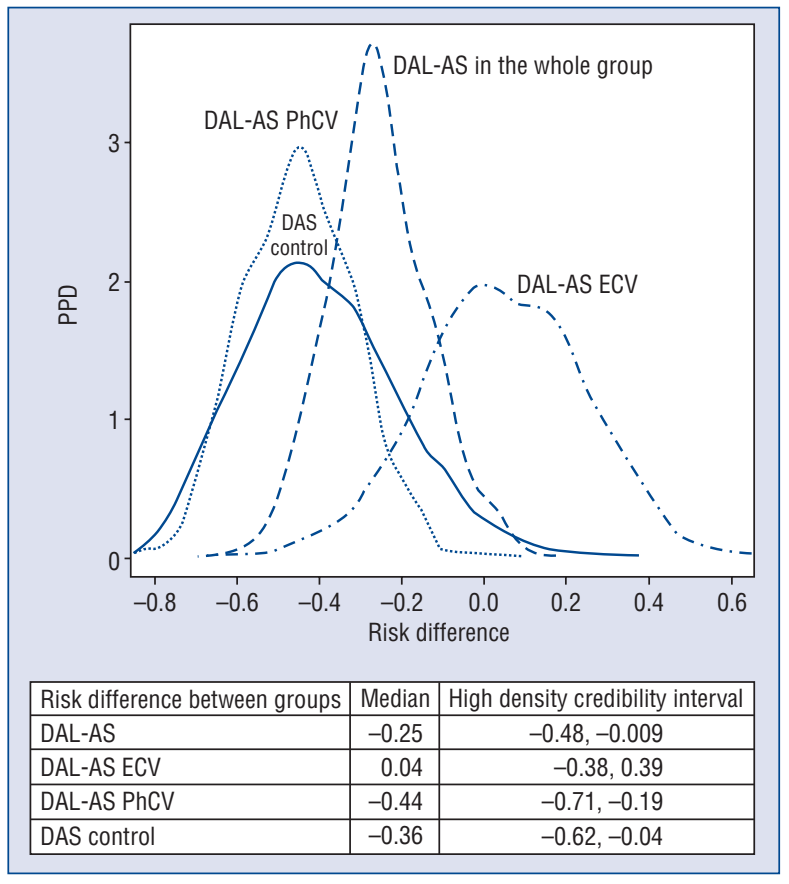

Figure 4. Curves disclosing the posterior probability density (PPD) of atrial fibrillation recurrence risk by means of a Bayesian perspective; DAL-AS ECV - density amiodarone long-amiodarone short electrical cardioversion; DAL-AS - density amiodarone long-amiodarone short; PhCV - pharmacologic cardioversion; DAS-control - density amiodarone short-control.

tient had overt manifestations of hypothyroidism). None of the patients under short-term amiodarone therapy exhibited limiting side effects.

Figure 4 shows the smoothed curves of the probability density function of $\emptyset$ (risk difference).
In density amiodarone long-amiodarone short (DAL-AS) ECV, the $\emptyset$ area is equiprobable to both sides of zero, which indicates no differences in the recurrence risk of arrhythmia. However, for DAL-AS pharmacological cardioversion (PhCV) as well as for DAS-control, the whole $\varnothing$ area is virtually located to the left of 0 , resulting in a clear risk reduction of AF recurrence. Moreover, for all groups in DAL-AS, the area $\varnothing$ is to the left in an intermediate location, and indicates a risk reduction, though of lesser magnitude. The average means values are shown at the bottom of Figure 4 .

\section{Discussion}

The clinical benefits of restoration and maintenance of SR in patients with persistent AF, including improvements in quality of life and functional capacity, as well as reduced morbidity and mortality, have been widely reported [1, 2, 12-16]. Diverse pharmacologic strategies directed toward long-term preservation of normal SR after reversion of persistent AF have been proposed, with modest antiarrhythmic efficacy and the incidence of limiting and even harmful adverse effects [17, 18]. Long-term amiodarone treatment is among the most effective treatments for promoting normal SR maintenance in patients with persistent AF [4-7]. However, the untoward side effects of the drug increase along with the duration of administration.

In this study, out of 51 patients, 35 (68.6\%) with the first-episode of persistent AF who recovered normal SR either by amiodarone alone, or by $\mathrm{ECV}$, and did not experience arrhythmia relapses during the initial 3-month treatment period, remained in normal SR after 18 months of follow-up. 
This was in contrast with the poor outcome documented in 8 of $9(88.9 \%)$ control patients, who developed AF relapses early in the follow-up period. Likewise, it is clear that long-term treatment is more effective than short-term treatment in patients regaining SR during the first 4-6 weeks of therapy ( $100 \%$ vs. $50 \%$ ). In a previous study, 45 of 56 patients $(83.6 \%)$ who experienced persistent $\mathrm{AF}$ and had their normal rhythm restored by oral amiodarone treatment, remained in SR at the 18-month follow-up [7]. In the present study, all patients under this condition retained SR at the end of the follow-up period, likely due to the small sample size. Further, long-term administration provides a high rate of rhythm control, but limiting side effects are more frequent as observed in 3 out of 27 patients in Group I (11.1\%) who developed clinically significant thyroid dysfunction and not seen in patients assigned to receive the placebo after the first 3 months of amiodarone treatment. Conversely, short-term amiodarone treatment is accompanied by a higher chance of AF relapses, but fewer or no serious side effects. The fact that amiodarone can be administered to patients even in the presence of structural heart disease and advanced heart failure is a significant additional advantage, given the contraindications of these conditions for other antiarrhythmic drugs.

No significant differences were found in outcomes for patients assigned to long-term and short-term amiodarone therapy, whose normal SR was restored by ECV, and those under short-term treatment, and whose normal SR was restored following the initial period of amiodarone administration.

Little information is available on the results of short-term amiodarone administration after conversion of the first episode of persistent AF. Channer et al. [19] achieved pharmacological reversion of the arrhythmia in $21 \%$ (26/123) of patients, although this study differs from our study in several aspects, and the reported results are not too different from those described here. However, these investigators used a relatively low loading dose of amiodarone ( $400 \mathrm{mg} /$ day for 2 weeks), and in addition, short-term amiodarone therapy $(200 \mathrm{mg}$ daily) lasted only 8 weeks, while long-term therapy was prolonged to 52 weeks. A year later, 20 out of 48 patients (42\%) who received short-term amiodarone were in normal SR, while the same antiarrhythmic effect was documented in 30 out of 48 patients (63\%) under long-term treatment. In marked contrast, normal SR persisted in only 2 out of 30 patients $(6.66 \%)$ not receiving amiodarone (control group). Limiting adverse effects were observed in $8 \%$ and $18 \%$ of patients receiving short-term and long-term amiodarone, respectively, and in 3\% in the control group.

Ahmed et al. [20] analyzed the rate of AF recurrences in patients who underwent transient (1 month) or continuous amiodarone treatment after reversion of recurrent episodes of persistent $\mathrm{AF}$, and restarted amiodarone administration in the first group whenever AF relapsed. After a mean follow-up of 25 months, the percentage of patients maintaining normal SR was $48 \%$ in those transiently treated with $200 \mathrm{mg}$ of amiodarone, and $62 \%$ in patients under continuous therapy with the same doses. However, the rate of AF recurrences was notably higher $(80 \%)$ in the transient group than in the continuous group (54\%; $\mathrm{p}<0.001)$. In this study, no significant differences were found in the incidence of adverse events that could be related to the duration of amiodarone treatment (19\% vs. $24 \%)$. Of note, mortality and hospitalization rates were significantly greater in patients with short-term amiodarone (53\%) compared with those receiving continuous therapy $(34 \%)(\mathrm{p}=0.002)$. The main implication of this study is that patients under amiodarone therapy for only 1 month after reversion of persistent $\mathrm{AF}$ show a higher incidence of arrhythmia recurrence, mortality, and cardiovascular hospitalizations. The main differences between the work of Ahmed et al. [20] and our study is that they included patients who had had AF relapses, the short-term treatment with amiodarone lasted only for 1 month, and AF relapses were reverted during follow-up.

Influenced by the latest guidelines and consensus, many cardiologists do not recommend systematic treatment with antiarrhythmic agents after the first episode of persistent AF, except in patients in whom the arrhythmia is accompanied by hemodynamic derangement or is highly symptomatic. In the absence of prophylactic treatment, the rate of relapses after $\mathrm{AF}$ conversion is high (more than $75 \%$ at 1 year after the initial crisis) [19]. This approach is partly supported by studies in which no significant difference was found in morbidity or mortality, with strategies directed to heart rate control or rhythm control $[7,21]$. However, this is conflicted by additional studies which report that SR preservation is associated with a lower risk of mortality and stroke, and improvement of functional capacity and quality of life $[12,13,22]$. 


\section{Does maintenance of SR during 3-month treatment with amiodarone revert atrial remodeling?}

Atrial remodeling in $\mathrm{AF}$ is induced by the rapid atrial rate and results in electrophysiological and structural changes that favor initiation and maintenance of arrhythmia [23]. Based on this concept, any antiarrhythmic treatment that seeks to maintain SR may prevent or even reverse atrial remodeling, which is a crucial factor to avoid $\mathrm{AF}$ relapses after reversion of the arrhythmia [10, 23]. Electrophysiological atrial remodeling is characterized by a shortening of repolarization, refractoriness dispersion, and slow impulse propagation. Abbreviation of atrial refractoriness is related to downregulation of $\mathrm{L}$-type $\mathrm{Ca}^{2+}$ currents resulting from intracellular calcium overload due to the rapid atrial rate [23]. This change leads to shorter atrial wavelengths, favoring the occurrence of multiple wavelet re-entries and thus increasing the susceptibility to AF and likelihood of AF maintenance [23, 24]. Additionally, increased atrial size may be important in this process since it may facilitate more re-entry circuits, and it is an important clinical predictor for $\mathrm{AF}$ development and perpetuation [25]. AF remodeling is not a permanent event, since even in persistent $\mathrm{AF}$, the remodeling is reversible, a fact that is of paramount importance in the management of patients with this form of AF. Indeed, conversion of AF to SR, either by electrical or pharmacologic means or after radiofrequency ablation, reduces atrial size [25, 26], and improves atrial contractility [27-29].

Disappearance of structural and functional changes generally lags behind the reversal of electrical remodeling [29, 30]. However, although reversal of electrical remodeling can be rapidly achieved [28, 29], the chance of $\mathrm{AF}$ recurrence may also depend on the underlying structural abnormalities [25]. Abbreviation of the P-wave and prolongation of the atrial refractory period, accompanied with reduced susceptibility to AF are the primary manifestations of reverse electrophysiological remodeling. These findings were reported only in AF patients who remain in SR and not in those who have AF recurrences. Furthermore, a high-resolution signal-averaged P-wave at 1 month and 3 months post cardioversion, has significantly decreased in those patients who remained in SR, whereas this has not been observed in those who relapsed [28]. It was postulated that the electrophysiological effects of amiodarone by itself might help to reverse atrial remodeling. In fact, in an experimental study in dogs by Shinagawa et al. [10], the administration of amiodarone after induction of atrial tachycardia remodeling by rapid pacing, reversed remodeling within days, although that rapid atrial pacing persisted during amiodarone therapy.

\section{Limitations of the study}

Although the small number of patients studied is a limitation, the detailed statistical analysis described in Results validates the conclusions of our study. Notwithstanding this fact, further larger studies are needed to better assess the safety and efficacy of the approach proposed in this study.

The ECGs performed during regular check-ups throughout the follow-up period, and at the end of the study, do not completely rule out the possibility of asymptomatic paroxysmal AF episodes. However, in the AFFIRM protocol as well as in the RECORD AF [7, 18], the success of evaluating rhythm control was based on single ECG recordings.

\section{Conclusions}

The findings of the current study indicate that if the first episode of $\mathrm{AF}$ is reverted with oral amiodarone therapy or ECV, and SR is maintained for at least 3 months, with amiodarone, more than 50\% of these patients will not experience AF relapses during the subsequent 15 months. Furthermore, this approach significantly reduces drug-related side effects. Patients who regain normal SR with amiodarone show a high rate of persistent long-term SR when the drug is administered for long periods.

\section{Conflict of interest: none declared}

\section{References}

1. Singh SN, Tang XC, Singh BN et al.; for the SAFE-T Investigators. Quality of life and exercise performance in patients in sinus rhythm versus persistent atrial fibrillation. J Am Coll Cardiol, 2006; 48: 721-730.

2. Singh BN, Singh SN, Reda DJ et al.; for the Sotalol Amiodarone Atrial Fibrillation Efficacy Trial (SAFE-T) investigators. Amiodarone versus sotalol for atrial fibrillation. N Engl J Med, 2005; 352: 1861-1872.

3. Subhaschini A, Gowda AS, Steinberg JS. Cardioversion of atrial fibrillation. Prog Cardiovasc Dis, 2005; 48: 88-107.

4. Roy D, Talajic M, Dorian P et al.; for the Canadian Trial of Atrial Fibrillation Investigators. Amiodarone to prevent recurrence of atrial fibrillation. N Engl J Med, 2000; 342: 913-920.

5. Galperin J, Elizari MV, Chiale PA et al. Efficacy of amiodarone for the termination of chronic atrial fibrillation and maintenance of normal sinus rhythm: A prospective, multicenter, randomized, controlled, double blind trial. J Cardiovasc Pharmacol Therapeut, 2001; 6: 341-350. 
6. Galperin J, Elizari MV, Chiale PA et al.; for the Grupo de Estudio de Fibrilacion Auricular con Amiodarona (GEFACA) Investigators. Pharmacologic reversion of persistent atrial fibrillation with amiodarone predicts long-term sinus rhythm maintenance. J Cardiovasc Pharmacol Therapeut, 2003; 8: 179-186.

7. Wyse DG, Waldo AL, DiMarco JP et al.; Atrial Fibrillation Investigation for Rhythm Management (AFFIRM) Investigators. A comparison of rate control and rhythm control in patients with atrial fibrillation. N Engl J Med, 2002; 347: 1825-1833.

8. Camm AJ. Safety considerations in the pharmacological management of atrial fibrillation. Int J Cardiol, 2008; 127: 299-306.

9. Shinagawa K, Li D, Leung TK, Nattel S. Consequences of atrial tachycardia-induced remodeling depend on the preexisting atrial substrate. Circulation, 2002; 105: 251-257.

10. Shinagawa K, Shiroshita-Takeshita A, Schram G, Nattel S. Effects of antiarrhythmic drugs on fibrillation in the remodeled atrium. Insights into the mechanism of the superior efficacy of amiodarone. Circulation, 2003; 107: 1440-1446.

11. Burstein B, Nattel S. Atrial structural remodeling as an antiarrhythmic target. J Cardiovasc Pharmacol, 2008; 52: 4-10.

12. Corley SD, Epstein AE, DiMarco JP et al. Relationships between sinus rhythm, treatment, and survival in the Atrial Fibrillation Follow-up Investigation of Rhythm Management (AFFIRM) Study. Circulation, 2004; 109: 1509-1513.

13. Atwood JE, Myers JN, Tang XC, Reda DJ, Singh SN, Singh BN. Exercise capacity in atrial fibrillation: A substudy of the Sotalol-Amiodarone Atrial Fibrillation Efficacy Trial (SAFE-T). Am Heart J, 2007; 153: 566-572.

14. Benjamin EJ, Wolf PA, D’Agostino RB, Silbershatz H, Kannel WB, Levy D. Impact of atrial fibrillation on the risk of death: The Framingham Heart Study. Circulation, 1998; 98: 946-952.

15. Vidaillet H, Granada JF, Chyou PH et al. A population-based study of mortality among patients with atrial fibrillation or flutter. Am J Med, 2002; 113: 365-370.

16. Stewart S, Hart CL, Hole DJ, McMurray JJ. A population-based study of the long-term risks associated with atrial fibrillation: 20 year follow-up of the Renfrew/Paisley study. Am J Med, 2002; 113: 359-364.

17. The AFFIRM First Antiarrhythmic Drug Substudy Investigators. Maintenance of sinus rhythm in patients with atrial fibrillation. J Am Coll Cardiol, 2003; 42: 20-29.

18. Camm AJ, Breithardt G, Crijns H et al. Real-life observations of clinical outcomes with rhythm- and rate-control therapies for atrial fibrillation. J Am Coll Cardiol, 2011; 58: 493-501.
19. Channer KS, Birchall A, Steeds RP et al. A randomized placebo-controlled trial of pre-treatment and short- or long-term maintenance therapy with amiodarone supporting DC cardioversion for persistent atrial fibrillation. Eur Heart J, 2004; 25: 144-150.

20. Ahmed S, Rienstra M, Crijns HJGM et al.; CONVERT Investigators. Continuous vs episodic prophylactic treatment with amiodarone for the prevention of atrial fibrillation. JAMA, 2008; 300: 1784-1792.

21. Roy D, Talajic M, Nattel S et al. Rhythm control versus rate control for atrial fibrillation and heart failure. N Engl J Med, 2008; 358: 2667-2677.

22. Connolly SJ, Crijns HJGM, Torp-Pedersen C et al.; for the ATHENA Investigators. Analysis of stroke in ATHENA: A placebo controlled, double-blind, parallel-arm trial to assess the efficacy of dronedarone $400 \mathrm{mg}$ BID for the prevention of cardiovascular hospitalization or death from any cause in patients with atrial fibrillation/atrial flutter. Circulation, 2009; 120: 1174-1180.

23. Schotten U, Verheule S, Korchhof P, Goette A. Pathophysiological mechanisms of atrial fibrillation: a translational appraisal. Physiol Rev, 2011; 91: 265-325.

24. Allessie M, Ausma J, Schotten U. Electrical, contractile, structural remodeling during atrial fibrillation. Cardiol Res, 2002; 54: 230-246.

25. Hagens VE, Van Veldhuisen DJ, Kamp O et al. Effect of rate and rhythm control on left ventricular function and cardiac dimensions in patients with persistent atrial fibrillation: Results from the Rate Control versus Electrical Cardioversion for Persistent Atrial Fibrillation (RACE) study. Heart Rhythm, 2005; 2: 19-24.

26. Reant P, Lafitte S, Jais $\mathrm{O}$ et al. Reverse remodeling of the left cardiac chambers after catheter ablation after 1 year in a series of patients with isolated atrial fibrillation. Circulation, 2005; 112: 2896-2903.

27. Sanders P, Morton JB; Kistler PM, Vohra JK, Kalman JM, Sparks PB. Reversal of atrial mechanical dysfunction after cardioversion of atrial fibrillation: Implications for the mechanisms of tachycardiamediated atrial cardiomyopathy. Circulation, 2003; 108: 1976-1984.

28. Pang H, Ronderos R, Perez Riera A, Femenia F, Baranchuk A. Reverse atrial electric remodeling: A systematic review. Cardiol J, 2011; 18: 625-631.

29. Kinobuchi O, Mitamura H, Shiroshita- Takoshita A et al. Temporal patterns of progression and regression of electrical and mechanical remodeling of the atrium. Int J Cardiol, 2005; 98: 91-98.

30. Choi JI, Park SM, Park JS et al. Changes in left atrial structure and function after catheter ablation and electrical cardioversion for atrial fibrillation. Circ J, 2008; 72: 2051-2057. 